\title{
The Beck effect is back, now in color: A demonstration
}

\author{
DAVID NAVON and RUTH KIMCHI \\ University of Haifa, Haifa, Israel
}

\begin{abstract}
It has been repeatedly found (e.g., Beck, 1982; Beck \& Ambler, 1973) that a tilted T is detected better than an $\mathrm{L}$ when presented on the background of a number of upright Ts, although in isolation both are discriminated from an upright $\mathrm{T}$ about equally well. We demonstrate that an analogous interaction between context presence and stimulus type can be obtained with color stimuli: A light green was discriminated from a dark green better than an olive brown was, yet that occurred only in the context of a background of three dark green disks. This indicates that the effects, both original and novel, probably are not due to anything peculiar to form perception, such as line arrangement or supraelement configurations. It is suggested that the presence of collateral background stimuli probably enables the perceptual system to capitalize on relative judgment involving contrasts or deviations from a presented anchor value.
\end{abstract}

The effectiveness of different stimulus properties for stimulus discrimination is not always uniform across situations. A prominent example is the sort of context effect known as the Beck effect. Disparity of line orientation (such as that between an upright $\mathrm{T}$ and a tilted $\mathrm{T}$ ) enables easy segregation between groups of elements, whereas differences in the spatial relationships between features (as between an upright $\mathrm{T}$ and an upright $\mathrm{L}$ ) do not (Beck, 1966, 1967). Likewise, a single tilted T is detected better than a single $\mathrm{L}$ when presented on a background of a number of upright Ts (Beck, 1972, 1974; Beck \& Ambler, 1972, 1973). However, when the task is to respond to a single stimulus, a tilted $\mathrm{T}$ and an $\mathrm{L}$ are responded to about equally accurately (e.g., Ambler \& Finklea, 1976; Beck, 1972, 1974; Beck \& Ambler, 1973).

This phenomenon has been attributed to several alternative sources (see the review in Kimchi \& Navon, 2000). Most accounts share two premises: (1) that a group of identical stimuli is perceptually different from the sum of the individual stimuli making it up and (2) that the discriminability of a stimulus embedded in a group of other ones is accounted for by the ease with which the stimulus is segregated from the group when presented adjacently. In a study designed to test those accounts (Kimchi \& Navon, 2000), we demonstrated that these premises are not necessary for obtaining the effect: It was observed even when, in the within-context condition, the background consisted of just a single upright $\mathrm{T}$ and even when the imperative stimulus was well segregated from the back-

We are grateful for helpful comments made by Jacob Beck, Marylou Cheal, Jim Enns, Trammell Neill, and Jeremy Wolfe, as well as for the assistance of Ronen Kasten and Ziziana Lazar. Correspondence concerning this article should be addressed to D. Navon, Department of Psychology, University of Haifa, Haifa 31905, Israel (e-mail: dnavon@psy.haifa.ac.il). ground by a large spatial separation. Since the effect was not observed with no background, we identified a single necessary condition for the occurrence of the effect, which we termed relative judgment.

The condition seems to be the following one. Whereas making a binary choice to a single stimulus involves absolute judgment (namely, a process that tries to match the stimulus with candidate memory representations), the task of responding to a stimulus in the context of other ones involves relative judgment (namely, a process that takes advantage of the properties of the neighboring sensory environment). Conceivably, attributes that are not particularly diagnostic for deciding which of the memory representations match a single stimulus might, nonetheless, be conducive to discrimination on the background of other stimuli (cf. Uttal, Baruch, \& Allen, 1995a, as opposed to Uttal, Baruch, \& Allen, 1995b), because the disparity between the collateral stimuli with respect to those attributes would be quite perceptible (cf. Duncan \& Humphreys, 1989; Enns, 1986; Nothdurft, 1991). For example, line orientation may not be more critical than line arrangement for identifying a single stimulus, yet disparity in orientation between two stimuli or more may be readily apprehended.

\section{The Focal Attention Hypothesis}

That the effect is obtained even when the context is minimal does not rule out the possibility that it is related to differential attention sensitivity to the two kinds of disparate letters conditions. The striking difference between a single-stimulus display and a display in which that stimulus is presented in the context of at least one background stimulus might be attributed to attention, in that it is only within the former display that a stimulus benefits from focal attention in the strict sense. This hypothesis typically has been coupled with a specific argument for the indis- 
pensability of focal attention. A prevalent thesis posits that the special difficulty in detecting disparity in line arrangement is due to its being a spatial relationship or a feature conjunction. Processing of those is believed to depend on focal attention - hypothetically, for binding their component features (see, e.g., Julesz, 1981, 1984, 1986; Treisman \& Gelade, 1980; Treisman \& Sato, 1990).

Such an account would be less compelling, however, if it were to be shown that the effect could be replicated with simple color stimuli. After all, there is nothing to bind in a color patch, since it does not contain any spatial relationship between features or any feature conjunction.

Below, we will elaborate on yet another good reason for trying to replicate the Beck effect with color stimuli.

\section{The Configuration Hypothesis}

The relative judgment account circumscribes the locus of the effect quite a bit, yet it does not entail a single process model. Relative judgment might rely, for example, on the effects of collateral background stimuli on the perception of the imperative stimulus. Alternatively, it might rely on emergent properties that arise from perceptual interactions between the disparate stimulus and the background ones.

A popular instance of the latter sort of account posits that the group of upright Ts configures with the disparate letter and does so in a differential manner, depending on the specific letter. According to this configuration hypothesis, the Beck effect is due to the fact that the tilted-Tupright-Ts configuration is less similar to a configuration consisting just of upright Ts than the L-upright-Ts configuration is. The term configuration, at least within that hypothesis, denotes an emergent form propertynamely, some supraelement pattern or a property of such a pattern that is given rise to by an interaction between segments of the elements (e.g., the wedge-like quasi-shape formed by the "legs" of an upright T and a tilted T).

Testing that hypothesis is hard, since there is no easy way to manipulate the extent of configurality. There is, however, an indirect way. If a similar effect could be demonstrated with stimuli in which the emergence of a differential configuration was implausible, that would cast some doubt on the hypothesis.

For that purpose, it would be instructive to obtain an effect analogous to the Beck effect with simple color stimuli. Although color is an integral composite of hue, saturation, and brightness, adjacent color patches do not configure, in the sense used in the hypothesis, as adjacent letters may. Nor do the component factors of adjacent color patches configure, as segments of adjacent letters may. Color can, of course, serve as a basis for spatial organization (such as distinguishing one pattern from another adjacent to it or superimposed on it), but the pattern emerging from that process is naturally some form and, as such, is indifferent to the color of the elements making it. When the perception of a specific color is affected by the context-a surrounding field or the colors of neighboring stimuli-this is typically ascribed to the influence of the context color on the perception of the target color (see, e.g., Jameson \& Hurvich, 1964; Walls, 1960). Thus, relative judgment of colors must be based on color contrast or color disparity in one way or another, and not on a perceived configuration.

\section{The Rationale}

If the Beck effect were to be replicated with color stimuli, for which the configuration account would be implausible, that would weaken a configuration account for the original Beck effect as well. Furthermore, that would cast some doubt on the version of the attentional hypothesis that attributes the effect to the dependence of processing feature conjunctions on focal attention. Although both hypotheses would still be viable as accounts of the original effect, the parsimony criterion would favor a more general hypothesis that could account for both variants of the effect alike.

To obtain a color variant, however, would require more than just picking three colors at random. The claim, it should be recalled, is that there is a fundamental difference between stimulus discrimination within context and without context, yet its support rests on a proof of existence. The original Beck effect does not hold, of course, for all sets of three-letter stimuli. Rather, the fact that it holds for some such sets indicates that stimulus discrimination is not governed by a universal, context-free process. The diversity in processing might surface and affect operational measures only in specific circumstances. The Beck effect, for example, might capitalize on the fortunate fact that whereas line orientation is not particularly diagnostic for identifying a single stimulus, disparity in line orientation is highly noticeable.

How does one go about looking for a comparably fortunate fact in color perception? Clearly, the colors should not be equidistant in terms of the dimensions most critical for relative judgment. We had a hunch that those, presumably, would be brightness and saturation. One of the disparate colors should be much more dissimilar to the background color than is the other disparate color in terms of, say, brightness.

That may or may not be enough. To be on the safe side, we tried to mimic another property of the set of stimuli used for obtaining the original Beck effect. The observed difference between line orientation and line arrangement could be somehow related to their different roles in defining categories of familiar shapes, such as letters. Whereas line orientation is used to characterize the canonical instance (e.g., an upright T), line arrangement is more or less common across instances (e.g., across Ts oriented in different angles). Thus, however dissimilar a tilted $\mathrm{T}$ is to an upright $\mathrm{T}$, their common name might impair any task of absolute judgment in which they fall in two different response categories. If that working assumption is valid, a color version of the Beck effect would have to use a triplet of colors $(a, b$, and $c)$ characterized by the following tenets: Color $b$ shares a hue name with Color $a$ but not with Color $c$, whereas the $a-b$ brightness disparity is greater than the $b-c$ brightness disparity. 
We generated a triplet that seemed to meet this criterion, according to our own judgment, ${ }^{1}$ as well as the brightness measures yielded by the luminance levels specified by the PCPaint software. The brightness differences were 72 for the $a-b$ color pair and 24 for the $b-c$ color pair. We then used that triplet in an experiment that was meant to parallel the procedure used to obtain the Beck effect.

Finally, we modeled the following experiment after two conditions in an experiment in which we had formerly obtained the Beck effect with forms (Kimchi \& Navon, 2000, Experiment 7).

\section{METHOD}

\section{Apparatus and Setting}

Stimulus presentation and data acquisition were controlled by an $\mathrm{O}^{2}$ Silicon Graphics computer. Stimuli were presented on the screen of a 15-in. computer display. Each subject sat in front of the display and responded by pressing on computer keyboard keys with the index fingers of the two hands. Viewing distance was about $100 \mathrm{~cm}$.

\section{Stimuli}

Each of the stimuli presented was a colored disk having a diameter of $8 \mathrm{~mm}$ (making $0.46^{\circ}$ of visual angle for a viewing distance of $100 \mathrm{~cm}$ ). The possible colors were light green, dark green, and brown (labeled $a, b$, and $c$, respectively). RGB values (weights of red, green, and blue in the additive mixture) were $(0,255,153),(0,102,52)$, and $(52,50,0)$ for $a, b$, and $c$, respectively. The fixation mark was a black dot having a diameter of $1 \mathrm{~mm}$, presented at the center of the screen.

Two display types were used in two different conditions - a singlestimulus display and a four-stimuli display. In the single-stimulus display, one disk was presented in any of the four quadrants of an imaginary $18 \times 18 \mathrm{~mm}$ matrix. In the four-stimuli display, a disk was presented on each of the quadrants of the imaginary matrix.
The distance between the centers of the disks measured $10 \mathrm{~mm}$ $\left(0.57^{\circ}\right)$ both horizontally and vertically, so that the side of the array measured $18 \mathrm{~mm}\left(1.03^{\circ}\right)$ and the euclidean distance between the center of each disk and the center of the screen was $7 \mathrm{~mm}\left(0.40^{\circ}\right)$. The disks within the matrix either were all of the same color (the one labeled $b$ ) or consisted of three of the color labeled $b$ and one disparate color (either the one labeled $a$ or the one labeled $c$ ) at either of the four matrix quadrants. The three possible displays in the four-stimuli condition are illustrated in Figure 1A. The three possible displays in the single-stimulus condition are illustrated in Figure 1B.

\section{Design and Procedure}

The subjects were tested individually in one experimental session. Each subject was run in two conditions meant to manipulate the presence of context. The conditions were administered in two different blocks - a without-context condition and a within-context condition. In the former condition, the subject was presented on each trial with a single-stimulus display, whereas in the latter condition, he or she was presented on each trial with a four-stimuli display. In both conditions, the subject was required to make a binary response discriminating a display of the sort labeled $b$ in Figure 1 from either of the other two sorts of display (labeled $a$ and $c$ in Figure 1). However, whereas in the without-context condition the instructions referred to discriminating between colors, in the withincontext condition the instructions were to tell whether or not there was a disparate colored disk in the display. ${ }^{2}$

Each block was preceded by the administering of conditionspecific instructions and consisted of 36 practice trials and 120 experimental trials. Across the experimental trials, each of the cells of the factorial design of the two within-block factors- namely, the three sorts of displays (labeled $a, b$, and $c$ in Figure 1) and the four possible quadrants - was presented with equal frequency in a random fashion.

Each trial started with a beep and a fixation mark that appeared for $500 \mathrm{msec}$. The mark was followed immediately by the stimulus display, which remained on the screen until either the subject re-

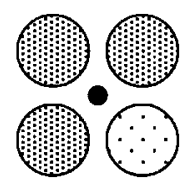

a

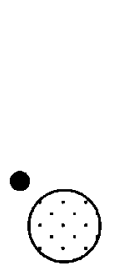

a

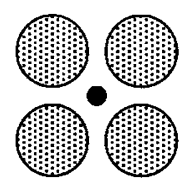

b
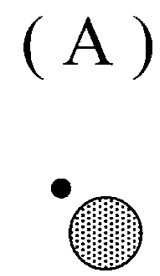

b

$$
\text { ( B ) }
$$

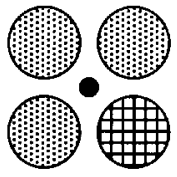

$\mathrm{c}$

Figure 1. Illustrations of the displays used in the experiment. Panel A illustrates the three sorts of displays used in the within-context condition. Panel B illustrates the three sorts of displays used in the without-context condition. Each of the display labels $(a, b$, and $c$ ) corresponds to the color label of the stimulus (in panel B) or of the disparate stimulus, when there is one (in panel A). The fixation point was actually much smaller than the dot representing it here. 
sponded or $2 \mathrm{sec}$ with no response had elapsed. The subsequent trial was initiated $1,200 \mathrm{msec}$ after that. The subjects responded by pressing on either of two response keys.

Block order and response key assignment were counterbalanced across subjects.

\section{Subjects}

Sixteen students at the University of Haifa served as subjects. All had normal or corrected-to-normal vision and participated in the experiment for part of their course credit.

\section{RESULTS}

Mean reaction times (RTs) for correct responses and percentages of errors for the three types of stimuli ( $a, b$, and $c$ ) as a function of the context manipulation are presented in Table 1.

Errors were scarce, and analysis of the accuracy data did not indicate any hint that RT effects might be due to a speed-accuracy tradeoff.

A two-factor repeated measures analysis of variance (ANOVA) performed on the RT data indicated a significant effect of stimulus type $[F(2,30)=15.74, p<.0001]$ and a significant interaction with the presence of context $[F(2,30)=7.11, p<.005]$. No significant main effect of context presence was found $[F(1,15)<1]$.

A separate ANOVA without Stimulus $b$ - the one from which both Stimulus $a$ and Stimulus $c$ were to be discriminated—revealed a similar pattern: A significant effect was found both for stimulus type $[F(1,15)=4.78$, $p<.05]$, and there was a significant interaction with the presence of context $[F(1,15)=12.93, p<.005]$. No significant main effect of context presence was found $[F(1,15)<1]$.

Analyses of simple effects of stimulus type within the context conditions indicated that the difference between Stimuli $a$ and $c$ was significant within context $[F(1,15)=$ $22.61, p<.0005]$, but not without context $[F(1,15)=$ $1.94, p=.184]$.

Thus, the effect of stimulus type seems to differ qualitatively between context conditions. The light green disk (labeled $a$ ) was responded to faster than the brown disk (labeled $c$ ) when presented within the context of the dark green disk (labeled $b$ ), but in isolation both were discriminated from the dark green disk about equally

Table 1

Mean Reaction Times (RTs) for Correct Responses (in Milliseconds) for the Three Sorts of Displays ( $a, b$, and $c$ in Figure 1) as a Function of the Presence of Context, With Percentages of Errors (PEs)

\begin{tabular}{|c|c|c|c|c|c|c|}
\hline \multirow[b]{3}{*}{ Condition } & \multicolumn{6}{|c|}{ Display } \\
\hline & \multicolumn{2}{|c|}{$a$ (Light Green) } & \multicolumn{2}{|c|}{$b$ (Green) } & \multicolumn{2}{|c|}{$c$ (Brown) } \\
\hline & RT & $\mathrm{PE}$ & $\mathrm{RT}$ & $\mathrm{PE}$ & RT & $\mathrm{PE}$ \\
\hline Without context & 558 & 0.6 & 576 & 3.4 & 548 & 0.3 \\
\hline Within context & 534 & 1.1 & 593 & 2.0 & 563 & 1.1 \\
\hline
\end{tabular}

Note-Each of the color names given in parentheses is the name of the stimulus (in the without-context condition) or of the disparate stimulus, if there is any (in the within-context condition). well. Even if the null effect without context was due to lack of statistical power, adding power would be likely to yield an effect that would go in the direction opposite to the one observed within context, to judge by the trend observed in the present data (see Table 1).

\section{DISCUSSION}

The present results illustrate that the Beck effect is not restricted to form discrimination, let alone to forms generated by configurations of line segments. It thus seems unlikely that the account of the effect is to be sought in anything peculiar to form perception.

The results can be straightforwardly interpreted in the following way. In the within-context condition, RT is affected by the dissimilarity of the disparate stimulus to the background ones (see, e.g., Cheal \& Lyon, 1994; Duncan \& Humphreys, 1989; Quinlan \& Humphreys, 1987). That is true for color stimuli as well (e.g., Nagy \& Sanchez, 1990). Specifically, the context of three dark green disks enables a discrimination between another dark green disk (the one labeled $b$ ) and either of the other disks (labeled $a$ or $c$ ) that employs contrasts between simultaneously presented colors or deviations from the anchor afforded by the background. The light green disk (labeled $a$ ) is easier to discriminate from the dark green disk than is the olive brown disk (labeled $c$ ), due to the greater brightness disparity between the light green and the dark green.

On the other hand, in the no-context condition, any brightness disparity cannot be sensed at all. To the extent that brightness differences retrieved from memory are nonetheless taken into account in absolute judgment, they cannot possibly be as critical as they are in relative judgment, since retrieved brightness is most probably fuzzier than sensed brightness.

By this account, for a discrimination done in the within-context condition, there was no resort to the same sort of absolute judgment as that required for a discrimination (actually, identification) done without context. Furthermore, it could not depend, in the case at hand, on perceiving a configuration, since colors do not configure in the sense that adjacent forms might. ${ }^{3}$ It must capitalize on the color disparity, when such exists. That disparity might exert its effect directly or indirectly: The response might be oriented to the disparity in itself, to the color pair having the disparity, or to the disparate stimulus judged against the surround. Whichever the case may be, a perceived configuration (in any nonloose sense) is not involved. By extension, it might not be necessary for the original Beck effect, since the latter can be accounted for without resorting to differential configuration. Although it is not impossible that each of the variants of the effect has its own specific account, maintaining that is clearly less general than positing a unified account.

Extending the Beck effect to the color domain casts some doubt also on another inference sometimes drawn from the original effect. The finding that discriminating by line arrangement is impaired by the addition of back- 
ground stimuli more than discriminating by orientation is (e.g., Beck \& Ambler, 1973) might be interpreted as indicating that the perception of line arrangement specially requires focal attention (cf. Cheal \& Lyon, 1992, 1994; Cheal, Lyon, \& Hubbard, 1991). Since orientation is believed to be a simple feature, whereas line arrangement involves a relationship between features, this has been regarded as evidence for the dependence of the perception of conjunctions (see, e.g., Treisman \& Gelade, 1980; Treisman \& Sato, 1990) or of the perception of spatial relationships (see, e.g., Julesz, 1981, 1984, 1986) on focal attention.

However, the present results demonstrate that a similar effect can be obtained for values of a single dimension that do not differ qualitatively. Although the color attribute-being an integral composite of hue, saturation, and brightness - is probably not as elementary as orientation, it is certainly not a conjunction of separable, independent attributes; nor is any particular color a conjunction of such attributes. An effect obtained by manipulating values on the same dimension cannot be meaningfully ascribed to a difference between simple attributes and attribute conjunctions.

It might be argued that the Beck effect is just a truncated example of visual search. Since discriminability typically affects the slope of RT set size functions (e.g., Cheal \& Lyon, 1994; Wolfe, 1998), its effect must be small, possibly negligible, when the number of distractors is zero. Ironically, in contrast with the prevalent finding in visual search studies, here the presence of "distractors" probably facilitated the response to the light green disk. But there is a yet more basic problem with this argument: the seemingly innocuous postulate that, on the abscissa, 0 differs from 1 in much the same way that 1 differs from 2 . That, however, is not always true. Nonpregnancy can hardly be described as zero-age pregnancy. Hence, the effects of pregnancy proper need not be mere extensions of the effects of pregnancy age. Psychological examples abound. It is especially important to examine an effect within the range $[0,1]$ when there is reason to think that the zero case corresponds to a different process. That seems to be the case with the Beck effect. The task in the blocked single-stimulus condition in this study is known by the name recognition. It is doubtful that this is a sort of visual search, since it lacks an essential hallmark of a typical visual searchnamely, the presence of distractors. That makes the process unable to employ any target-distractor match or disparity that we can call relative judgment. Contrasting stimulus effects in the single-stimulus condition and in the within-contextcondition is, thus, a between-tasks comparison. Whether or not the interaction between stimulus and condition is caused by the very same cause as that of the interaction of stimulus with number of stimuli in the latter condition is as yet a matter of conjecture.

One might contend that the phenomenon demonstrated here is not necessarily general. The simple an- swer is that the experiments are meant just to be illustrative, as were the experiments that served to demonstrate the original Beck effect. For that purpose, we tried to mimic two properties of the stimuli used to obtain the original effect. Accordingly, we chose a background color (Disk $b$ ) that met two conditions: being more dissimilar from one disparate color (Disk $a$ ) than from the other one (Disk $c$ ) on a dimension that would affect relative judgment and sharing hue name with the former, but not with the latter. Either of these conditions or both may be necessary. That cannot be determined from the results of the present study. Our objective was just to provide a demonstration of existence. Occam's razor prescribes that the burden of proof is now on proponents of the configuration hypothesis, the focal attention hypothesis, or, for that matter, any other hypothesis that resorts to mental entities peculiar to form perception.

\section{REFERENCES}

Ambler, B. A., \& FinkleA, D. L. (1976). The influence of selective attention in peripheral and foveal vision. Perception \& Psychophysics, 19, 518-524.

BECK, J. (1966). Effects of orientation and of shape similarity on perceptual grouping. Perception \& Psychophysics, 1, 300-302.

BeCK, J. (1967). Perceptual grouping produced by line figures. Perception \& Psychophysics, 2, 491-495.

BECK, J. (1972). Similarity grouping and peripheral discriminability under uncertainty. American Journal of Psychology, 85, 1-19.

BECK, J. (1974). Relation between similarity grouping and peripheral discriminability. Journal of Experimental Psychology, 102, 11451147.

BECK, J. (1982). Textural segmentation. In J. Beck (Ed.), Organization and representation in perception (pp. 285-317). Hillsdale, NJ: Erlbaum.

BECK, J., \& AMBLER, B. (1972). Discriminability of differences in line slope and in line arrangement as a function of mask delay. Perception \& Psychophysics, 12, 33-38.

Beck, J., \& AMBLER, B. (1973). The effects of concentrated and distributed attention on peripheral acuity. Perception \& Psychophysics, 14, 225-230.

CheAl, M. L., \& LyON, D. R. (1992). Benefits from attention depend on the target type in location-precued discrimination. Acta Psychologica, 81, 243-267.

CheAl, M. [L.], \& LyON, D. R. (1994). Allocation of attention in texture segregation, visual search, and location-precuing paradigms. Quarterly Journal of Experimental Psychology, 47A, 49-70.

Cheal, M. [L.], Lyon, D. R., \& Hubbard, D. C. (1991). Does attention have different effects on line orientation and line arrangement discrimination? Quarterly Journal of Experimental Psychology, 43A, 825-857.

Duncan, J., \& Humphreys, G. W. (1989). Visual search and stimulus similarity. Psychological Review, 96, 433-458.

EnNs, J. (1986). Seeing textons in context. Perception \& Psychophysics, 39, 143-147.

JAMESON, D., \& HURVICH, L. M. (1964). Theory of brightness and color contrast in human vision. Vision Research, 4, 135-154.

JulEsZ, B. (1981). Textons, the elements of texture perception and their interactions. Nature, 290, 91-97.

JuLESZ, B. (1984). A brief outline of the texton theory in human vision. Trends in Neuroscience, 7, 41-45.

Julesz, B. (1986). Texton gradients: The texton theory revisited. Biological Cybernetics, 54, 245-251.

Kimchi, R., \& NAVON, D. (2000). Relative judgment seems to be the key: Revisiting the Beck effect. Journal of Experimental Psychology: Human Perception \& Performance, 26, 789-805.

NAGY, A. L., \& SANCHEZ, R. R. (1990). Critical color differences de- 
termined with a visual search task. Journal of the Optical Society of America A, 7, 1209-1217.

Nothdurft, H. C. (1991). Different effects from spatial frequency masking in texture segregation and texton detection tasks. Vision Research, 31, 299-320.

Quinlan, P. T., \& HuMPhreYs, G. W. (1987). Visual search for targets defined by combinations of color, shape, and size: An examination of the task constraints on feature and conjunction searches. Perception \& Psychophysics, 41, 455-472.

Treisman, A., \& Gelade, G. (1980). A feature integration theory of attention. Cognitive Psychology, 12, 97-136.

Treisman, A., \& SATO, S. (1990). Conjunction search revisited. Journal of Experimental Psychology: Human Perception \& Performance, 16, 459-478.

Uttal, W. R., Baruch, T., \& Allen, L. (1995a). Combining image degradations in a recognition task. Perception \& Psychophysics, 57, 682-691.

Uttal, W. R., Baruch, T., \& Allen, L. (1995b). The effect of combinations of image degradations in a discrimination task. Perception \& Psychophysics, 57, 668-681.

WALls, G. L. (1960). Land! Land! Psychological Bulletin, 57, 29-48. Wolfe, J. M. (1998). Visual search. In H. Pashler (Ed.), Attention (pp. 13-73). Hove, U.K.: Psychology Press.

\section{NOTES}

1. We validated our judgment by obtaining, from various subjects, verbal responses specifying the description of the color of any of these three colors and analyzing them for common hue names. Twenty-nine subjects out of 32 mentioned a common hue name in their verbal descriptions of the two colors we had judged as sharing a hue name (the ones labeled $a$ and $b$ ), whereas only 21 subjects out of 49 did it for the other pair (the two colors labeled $b$ and $c$ ). The modal names given were light green for $a$, green for $b$, and brown for $c$.

2. Previous research (Kimchi \& Navon, 2000) has ruled out the possibility that the difference between without-context and within-context conditions, when present, is due to instructions.

3. It is possible to contend that adjacent colors "configure" in some other sense-by giving rise to symmetry/asymmetry or homogeneity/ heterogeneity. These, however, are basically dichotomous properties. It is hard to believe that the two kinds of disparate colors gave rise to differential asymmetry or differential heterogeneity. Even if they did, the cause probably would have been differential disparity.

(Manuscript received June 17, 2002;

revision accepted for publication September 3, 2002.) 